\title{
Interactive Robot for Automated Question and Answer System
}

\author{
Vijay A. Kotkar ${ }^{1}$, Muskan S. Sahay ${ }^{2}$, Manasi S. Kate ${ }^{3}$, Ashwini K. Mante ${ }^{4}$, Prachi S. Kaspate ${ }^{5}$ \\ ${ }^{1}$ Faculty, JSPM's Rajarshi Shahu College of Engineering, \\ 2,3,4,5 Student, JSPM's Rajarshi Shahu College of Engineering.
}

Article History: Received: 10 November 2020; Revised: 12 January 2021; Accepted: 27 January 2021; Published online: 05 April 2021

\begin{abstract}
This paper aims in developing an intelligent interactive robot with multi-functions which provides entertainment and companion. To obtain the information accurately, we have used speech recognition to perform the operations. For the Robot behavior, planning, interactions with the voice assistant and interactions with the user the various speech recognition results are applied. The robot has a simple design. In this study, we have used the microphone for speech recognition. In addition, we have used room automation, notice display using voice message of the intelligent interaction between human and robots.
\end{abstract}

Keywords: Interactive intelligent robot, notice board, speech recognition, human-robot interaction.

\section{Introduction}

Most of the fields in the robotics have created robots that functions on one specific task(goal) instead of creating a multi-functional robot that can provide effectiveness in the working of more than a single job.Interactive robot is a developing robot engine and uses cognitive technology. Human robot interaction has become a big challenge which needs to be deal with to introduce the human robot interaction into our society.In this proposed system, an interactive robot that combines notice board module, question and answer module automation module, recording etc. The robot must be capable of quick responses and timely responses so that there can be a sustainable interaction between the robot and the user. Such socially interactive robot plays an important role in the human robot interactions. Some functions are provided so that the robot can act as the assistant to the human users through interactions. [2] The implemented system uses Raspberry pi 3. Raspberry pi 3 has Bluetooth, it has Wi-fi, and it has more powerful CPU/GPU pair. The power consumption, network speed, performance gain is more as compared to Raspberry pi 2.[3]It stores the voice signals using Audacity, and the sampling is done at the rate of 16000 samples per second. Each speech signal will be divided into windows of certain milliseconds depending upon the total number of samples. Later the samples in the windows are matched to recognize the particular voice. Due to the changes in the recording locality there may be differences in the frequencies, therefore large number of coefficients are calculated for each user. Using the same technique the notice board will be implemented that will respond only to the voices of verified high authority people. Speechto-Text will be used to fetch the answers of the technical questions asked by the user, the system will fetch the answers from Wikipedia.The robot will be connected by the cloud so that it can perform operations from web. In addition, the important information can also be mailed to the user and for doing so we will be using SMTP (Simple Mail Transfer Protocol). It is the standard protocol that is used for mail services on a TCP/IP network. This will enable the transmission and delivery of email over the Internet. After performing an action o sending the mail successfully the system will give the acknowledgement through the speaker. Robot will also be able to read the recent mails if asked by the user. User's email address will already be stored in the system that will allow the robot to automatically fetch the information regarding any new mails or send any mails required. For operating the electrical appliances such as light and fan in the classroom can be operated using voice commands and sensors which uses automation. When the user will choose the option of switching the lights/fan on, the system will first check the current state of the appliance and then if the desired function is possible then the same will be implemented and the acknowledgement will be given through the speaker attached. Memory card has been added to the robot so as to record the lecture as per the requirement of the user and that memory card can be retrieved later to listen to the lecture by the students. The size of memory card will be according to the length of the lecture to be recorded an may vary depending upon the requirement. The whole system will be connected by the help of Raspberry pi 3. It will connect with all the components of the system and enable a proper communication between them. It includes $802.11 \mathrm{n} \mathrm{Wi-fi}$ and Bluetooth 4.0. It has 4 USB ports one of will be used to connect the external memory device and a power supply will also be connected to the board for continuous functioning of the system. Different features are coupled together so as to form a complete automated classroom system. 


\section{Literature Survey}

This section presents a brief discussion about recent techniques in the field of Human Robot Interaction. Ayanna Howard et.al. [1] Most of the human robot interactions are conducted in a laboratory or some are even conducted with the presence of the actual robot. These experiments can be more time consuming. In this paper a solution to overcome this problem is stated by using a platform in which the users can remotely interact with a robot via video based framework.

I HameemShanavas et.al. [2] In this paper Raspberry Pi engine is used to develop a interactive personal assistant robot.

Robert S. Gutzwiller et.al. [3] This paper a interactive method IML for human input to decrease manpower, machine learning techniques that are gaining visibility.

Zhe Chen et.al. [4] In this paper an experiment for a better investigation of the factors to determine user engagement level is designed. It gives how humans are engages with the robots and what humans expects from the robots.

J. Calzado et.al. [5] This paper has proposed a SAMI robot architecture which extends the reactive paradigm by adding the interactive layer.

Satoshi Hoshino et.al. [6] This paper states a robot which helps humans to guide towards a proper direction. It is done with the help of multiple mobile robots to guide the humans. The robots givesthe exact moving direction to the humans. Suppose the direction given by the robot is correct then the human is attracted by the robots.

Mike Ligthart et.al. [7] In this paper the several studies are done and investigated in case of a child robot interaction. To be ware of the child's expectations, to educate the child with good habits and also to acknowledge the robots are the three main strategies

Saad Ahmed Rahat et.al. [8] This paper uses Python speech voice recognition module and Raspberry Pi so that anyone can control the system using their voice commands.

SeverinsonEklundh et.al. [9] This paper explores how a robot's social status influences participant response and the limits of that response. Extent to which social status may play out in a robot that is not human-like in appearance. It give the social characteristics of robot with respect to interactive behavior with agents.

Amy Eguchiet. al. [9] This paper remotely interact with a socially interactive robot. To establish a relationship between believability and the actual presence of the robot this interactive robot is designed. This robot can be remotely controlled by the users.

Jian-Hao Hong et. Al. [10] This paper stated a robot which can be controlled with a varying supply voltage. The servo motors are used along with the Raspberry Pi engine. This robot can be used as a personal assistant robot.

KosukeTsujinoet. al. [11] In this paper approach for personalized therapies for social and behavioral learning. Children will learn interaction steps in a more systematic way while they are not overwhelmed.

Seiichi Kumagaiet. al. [12]In this paper, a way to improve the interactive cooperation between the users and various service robots is stated. The main disadvantage is that Needs improved efficiency. It uses HRI Interface.

P.R. Wumanet. al. [13] This paper uses the Machine learning techniques in exploring how to team the humans together effectively.

Q. Chen et. al. [14] Various factors like user engagement level, and to engage the students in the classroom are stated in this paper. Using this paper more advanced and emotionally intelligent robot can be implemented, which can detect various user aspects.

L. E. Parker et. al. [15] In this paper it corrects its plans whenever the human deviates from the predicted explicable plan. The main advantage of this paper Mobile, autonomous robot pet application. The drawback is as follows Does not use specific algorithms. It uses cybernetics and HRI.

M. J. Martric et.al. [16] In this paper it demands in reducing the mental health and attention switching. It does so by centering the overall interaction in the shared workspace. The advantage of this is that it can switch the interactions in a shared workspace

M. Mountz et.al. [17] This paper uses MANUSERV technique for task planning for humans. This robot can be used in agricultural fields and in planning agricultural environments.

K. Dautenhahn et.al. [18] In this paper various Robots to guide the Humans are stated by using Interactive Multiple mobile robots. It uses Co-ordinate system but cannot guide multiple people in the same time. It provides the local guidance formation.

A. Steinfeld et.al. [19]This paper focuses upon the Child expectation management by inspecting the ChildRobot interaction. It helps children's expectation management effectively. It is implemented using Child-robot interaction (CRI), Intrinsic Motivation Inventory (IMI).

David P.Anderson et.al. [20] This paper gives the information about the interaction between children and the robots. The study using this robots states about the children suffering with ASD. It helps children to live and enjoy their childhood. 


\section{Proposed Model}

This system consists of a 6 modules in which we perform following tasks:

1. Using speech recognition attendance monitoring

2. Home/office/college automation

3. Question and answer system

4. Using voce email operations

5. Using voice any message display to notice board

6. Voice recording

The main purpose of this system is that the improvement in human and robot interaction. The system work flow is in the following manner- Initially the voice signal from the human source is acquired by the web cam present along with the microphone which is inbuilt. These signals then get converted to a form that is understandable by the controller. The controller then searches for the actions performed by the user and the response which needs to be replied to the commands given by the user. Once the response if found by the controller the result is to be given by the speaker as the output. There are two output mediums used in this paper. One is the Screen and other is the speaker. This whole process of conversion is implemented using various voice interaction and voice recognition techniques. We have performed speech recognition for attendance monitoring system and for voice based notice board.

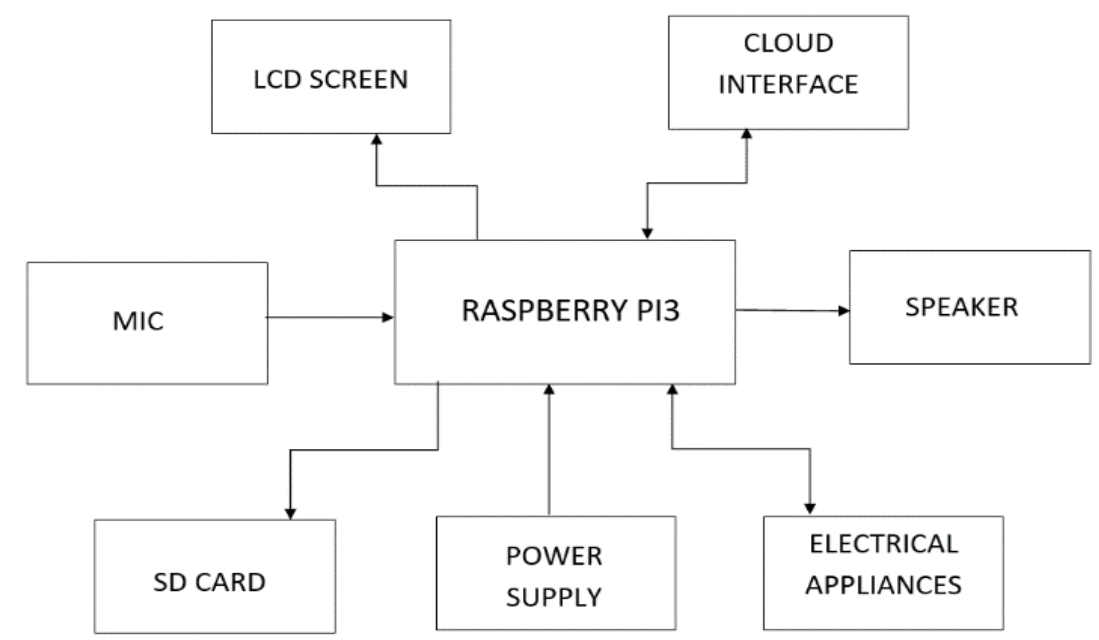

Figure 1. Block diagram

Figure 1 shows the components of our system and the interaction between them or how they are connected. Raspberry pi 3 is used as the main unit where all the other components are connected. LCD Screen is used to display the notice. Speaker is used as an output device where the answer will be given. Mic is used as an input device for all the interactions to take place between the system and the user. SD cared is used to store the recorded lectures in audio format.

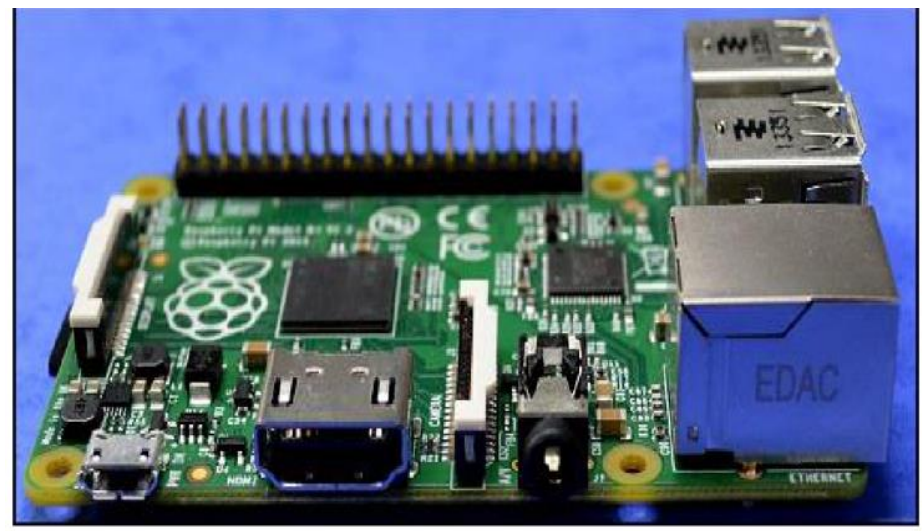

Figure 2. Raspberry Pi 3 


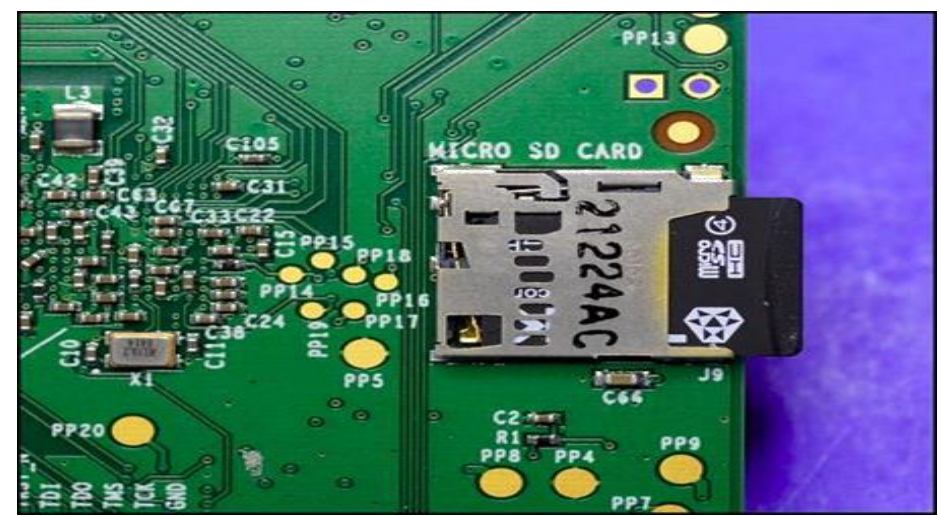

Figure 3. SD Card slot
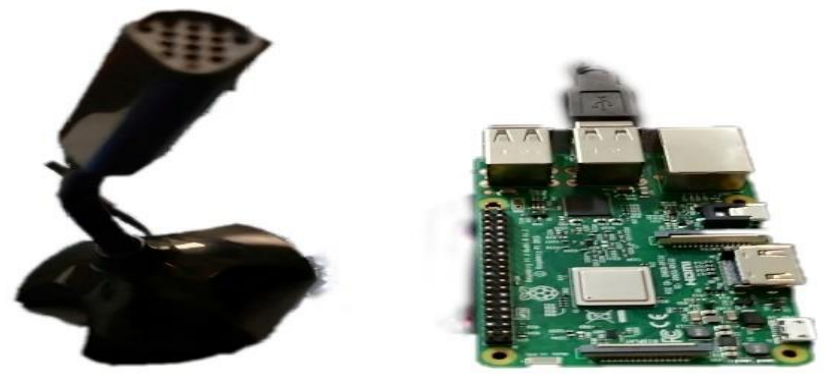

Figure 4. MIC and Raspberry pi

Conversion of Frequency to Mel scale:

$$
M(f)=1125 \ln (1+f / 7 !
$$

Conversion from Mels to frequency:

$$
M^{-1}(m)=700\left(\exp \left(\frac{m}{1125}\right)-\right.
$$

The Discrete Fourier Transform of the frame is done by using the following:

$$
s_{i}(k)=\sum_{n=1}^{N} s_{i}(n) h(n) e^{-\frac{j 2 \pi k n}{N}} 1 \leq k \leq
$$

$\operatorname{Here} h(n)$ is an $N$ sample long analysis window.

\section{Advantages}

1. Circuit complexity Reduced.

2. It can be used as virtual assistant.

3. Helpful to operate in colleges or schools.

\section{Results and Discussion}

As per our proposed system we build interactive robot. Using such robot we perform multiple tasks such as attendance monitoring, Home/office/college automation, Question and answer system, using voice email operations, using voice any message display to notice board and Voice recording. The results are shown below: 

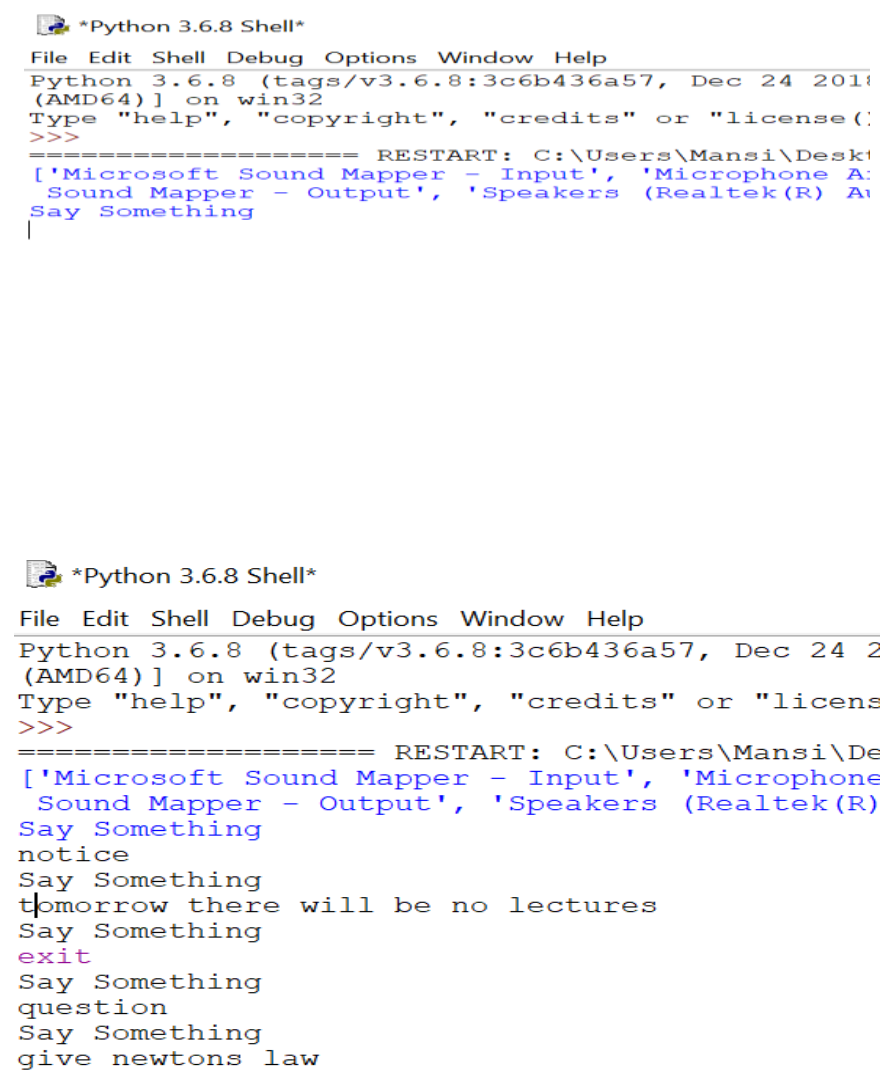

\section{Conclusion}

The interactive robot is a system developed for classrooms. The system does all the required work as per the need of the user. The system provides answers to all the technical questions as proposed. It is a fully automated system that can implement the electrical appliances and the notice on the screen as discussed. The teachers now don't have to worry about taking attendance by calling out the roll numbers of the students as the attendance will be marked by voice recognition of the students. The system/Robot will be completely classroom based. Much work of the faculty will be minimized by this voice interactive robot. Voice based notice board is provided for teachers who want to pass any notice to the students of the respective class. The lecture is recorded by the system by giving command so that the students can refer those recorded lectures in future. The system turns on/off the lights and fan of the classroom once the students have left the class. The workload of the teachers will reduce to some extent. The increased use of user and family friendly robots, the voice interaction based robots are most helpful and has many various applications. The robot that we have presented in this paper gives the user an interactive workspace to the user and reduces the workload by assisting him in a virtual environment.

\section{References}

1. Robot and Human interactive Communication, 2007. RO-MAN 2007. The 16th IEEE International Symposium on, pp. 872-877, IEEE, 2007.

2. Amy Eguchi and Hiroyuki Okada, Learning with social robots-The world robot summit's approach, Integrated STEM Education Conference (ISEC) 2018.

3. Felix Grasser, Aldo D'Arrigo,SilvioColombi, and Alfred Rufer., “ Joe: A mobile, inverted pendulum. "Laboratory of Industrial Electronics, Swiss Federal Institute of Technology Lausanne, EPFL, Switzerland.

4. David P.Anderson, "nBot Balancing Robot” SMUC., 14 Sep. 2013. 
5. Jian-Hao Hong, Dept. of Power Mech. Eng., Nat. Tsing Hua Univ., Hsinchu, Taiwan, “Analysis and design of a passive steering mechanism for a pedaled, self-balanced, personal mobility vehicle" in System Integration(SII), 2013, IEEE/SICE International Symposium.

6. Prof. YogeshRisodkar, Ganesh Shrisath, MonaliHolkar and MayurAmle, "Designing the selfbalancing platform(Segway)," Electronics and Telecommunication Department, SIEM, IJSETR, vol 4, issue 9, Sept.,2015.

7. BrainslavPopovie, EdvinPakoci, NiksaJakovljevic, "Voice Assistant application for the Serbian Language,”. 23rd Telecommunication forum TELFOR 2015.

8. Anurag Mishra1, Pooja Makula2, Akshay Kumar3, Krit Karan4 and V. K. Mitta15, Indian Institute of Information Technology, “A voice controlled personal assistant robot,",2015 International Conference on Industrial Instrumentation and Control (ICIC).

9. Seiichi Kumagai, Tomako Sato and Hiroki Sano, Graduate School of Industrial Technology, Advanced Institute of Industrial Technology, 110-40 Higashi-Ohi, Shinagawa-Ku, Tokyo, Japan," Cooking assistant service utilizing an interactive robot,", System Integration(SII), 2017 IEEE/SICE International Symposium.

10. KosukeTsujino, Shinya Lizuka and Yusuke Nakashima, Res. Labs., NTT DOCOMO, Inc., Yokosuka, Japan, "Speech Recognition and Spoken Language Understanding for Mobile Personal Assistants: A case Study of "ShabetteConcier",", Mobile data management(MDM), 2013 IEEE 14th International Conference.

11. P. R. Wurman, R. D'Andrea, and M. Mountz, "Coordinating Hundreds of Cooperative, Autonomous Vehicles in Warehouses,” AI Magazine, vol. 29, no. 1, pp. 9-19, 2008.

12. S. Hoshino and K. Maki, "Motion Planning of Multiple Mobile Robots Based on Artificial Potential for Human Behavior and Robot Congestion," Distributed Autonomous Robotic Systems, pp. 311-324, 2016.

13. S. Hoshino and K. Maki, "Safe and Efficient Motion Planning of Multiple Mobile Robots based on Artificial Potential for Human Behavior and Robot Congestion," Advanced Robotics, vol. 29, no. 17, pp. 1095-1109, 2015.

14. P. K. C. Wang, "Navigation Strategies for Multiple Autonomous Mobile Robots Moving in Formation,” Journal of Robotic Systems, vol. 8, no. 2, pp. 177- 195, 1991.

15. Q. Chen and J. Y. S. Luh, "Coordination and Control of a Group of Small Mobile Robots," IEEE International Conference on Robotics and Automation, pp. 2315-2320, 1994.

16. L. E. Parker, "On The Design of Behavior-Based Multi-Robot Teams," Advanced Robotics, vol. 10, no. 6, pp. 547-578, 1996.

17. M. J. Mataric, "Behavior-Based Control: Examples from Navigation, Learning, and Group Behavior," Journal of Experimental and Theoretical Artificial Intelligence, vol. 9, no. 2-3, pp. 323-336, 1997.

18. T. Balch and R. C. Arking, "Behavior-Based Formation Control for Multi-Robot Teams," IEEE Transactions on Robotics and Automation, vol. 14, no. 6, pp. 926-939, 1998.

19. Felix Endres, J"urgen Hess, J"urgen Sturm, Daniel Cremers, and Wolfram Burgard, "3-D Mapping With an RGB-D Camera," IEEE Transactions on Robotics, Vol. 30, No. 1, February 2014.

20. ZviGalil, Giuseppe F. Italiano, "Data Structures and Algorithms for Disjoint Set Union Problems," Journal ACM Computing Surveys Archive, Vol. 23 Issue 3, Sept. pp. 319-344, 1991.

21. J. Li, W. Ju, and C. Nass, "Observer perception of dominance and mirroring behavior in humanrobot relationships," Proceedings of the Tenth Annual ACM/IEEE International Conference on Human- Robot Interaction, pp. 133-140, 2015.

22. J. Li, R. Kizilcec, J. Bailenson, and W. Ju, "Social robots and virtual agents as lecturers for video instruction,"Computersin Human Behavior, vol. 55, part B, 1222-1230, Feb.

23. Liu, Jianshen, Yin, Baoyong, Liao, Xinxing, "Robot self-localization with Optimized Error Minimizing for Soccer Contest. J. Comput. 6(7), 1485-1492, 2011.

24. Liu, B., Fan, J., Zhou, J., Li, K., Xie, Y., "A Self-localization Method through Pose Point Matching for Autonomous Soccer Robot based on Omni-vision," The 9th International Conference on Electronic Measurement and Instruments, pp. 246-249, 2009.

25. Lauer, M., Lange, S., Riedmiller, M., "Calculating the Perfect Match: an Efficient and Accurate Approach for Robot Self-localization," RoboCup 2005: Robot Soccer World Cup IX, vol.4020 of the series Lecture Notes in Computer Science, pp. 142-153.

26. Heinemann, P., Haase, J., Zell, A., "A Combined Monte-Carlo Localization and Tracking Algorithm for RoboCup," Proceedings of the 2006 IEEE/RSJ International Conference on Intelligent Robots and Systems, pp. 1535-1540, 2006. 